\title{
Efficacy of arthrocentesis with injection of hyaluronic acid in the treatment of inflammatory-degenerative disease of temporomandibular joint
}

\begin{abstract}
Enas Abdulsattar Abdulmaged (1), Thair Abdul Lateef ${ }^{(2)}$
https://doi.org/10.26477/jbcd.v33i1.2920

ABSTRACT

Background:Temporomandibular joint (TMJ) problems refer to a group of heterogeneous pain and dysfunction conditions involving the mastication, reducing life quality of the patients. Arthrocentesis is a simple and less invasive surgical method for the treatment of inflammatory-degenerative disease than other conservative procedures and better than arthroscope. This clinical study aimed at evaluating the benefit of arthrocentesis with injection of hyaluronic acid in the management of inflammatory-degenerative disease of the TMJ.

Material and methods: Eighty consecutive patients were enrolled in this study with pain symptoms of TMJ, insufficient masticatory efficiency during function and limitation of mouth opening; they were assessed with clinical examination and approved with computed tomography scan. Arthrocentesis was done by inserting 18 gauge needles in the upper joint compartment, lavage by normal saline solution and at the end of the procedure $1 \mathrm{ml}$ of hyaluronic acid (HA) was injected. Intensity of the TMJ pain and masticatory efficiency was analyzed by visual analog scale (VAS), maximum mouth opening (MMO) was assessed by a ruler. All the assessed parameters were measured before the procedure then 1 and 4 months later.

Results: During 5 months follow-up, comparison of the obtained results showed reduction in pain at chewing and rest $87.5 \%$, improvement in mouth opening $100 \%$ and significant improvement in masticatory efficiency $87.5 \%$ of patients.

Conclusion: The procedure of arthrocentesis with Sodium Hyaluronate injection, used in patients who suffered from inflammatorydegenerative disease (IDD), showed therapeutic benefits, simplicity, safety, patient satisfaction, lack of significant side effects and complications.
\end{abstract}

Key words: Arthrocentesis, inflammatory-degenerative disease, hyaluronic acid. (Received: 2/1/2018, Accepted: 11/2/2018)

\section{INTRODUCTION}

Temporomandibular joint disorders is an umberella term covering pain and dysfunction of TMJ and represents therapeutic challenge in our maxillofacial department. ${ }^{(1)}$ Generally, the manegment of TMJ dysfunction depends on the criteria of TMJ condition and is based on the position and shape of the TMJ disc which is described by Dworkin and LeResche. The debate among scientists continues surrounding accompanying factor in tmj. ${ }^{(2)}$ All these disorders make several of suffering represented by jaw pain, mastication efficiency, limited jaw movement and TMJ destruction. ${ }^{(3)}$

Traditional non invasive approaches were applied to manegment of TMJ OA, physical therapy, occlusal splints acupuncture, pharmacological, injection of steroids and recently the injection of hyaluronic acid (HA) injection.

(1) Senior of Oral and Maxillofacial Surgery in Al-Wasity Teaching Hospital. Baghdad -Iraq, Ministry of Health.

(2) Assistant Professor in Oral and Maxillofacial Surgery Department, Collage of Dentistry, University of Baghdad.

Corresponding email, drenas999@yahoo.com
TMJ arthrocentesis is a minimally invasive technique, less expensive and simple with low morbidity used for flushing out TMJ that is done by double access to upper joint space. ${ }^{(4)}$

Its application enhances jaw function and achieves pain relief in patients with restricted mouth opening. ${ }^{(5,6)}$. HA infiltration which is polysaccharide of the family of glycosaminoglycans becomes an attributed option for the management and reliefing symptoms in the clinical setting. ${ }^{(7)}$

This leads to the progressive expansion of potential clinical indications for the use of combined technique arthrocentesis which provides expansion of joint space and washing out intra articular inflammatory mediator and carbolytes coupled by Hyaluronic acid (HA) injection to enhance joint lubrication reducing joint friction and replacemrnt of synovial fluid in TMJ. ${ }^{(8)}$

Namely, the effectiveness of joint lavage may be manifested by releasing the articular disc and breaking the adhesion and the adherence between disc surface and mandibular fossa thus increasing mouth opening.New era of utilization of Hyaluronic acid in TMJ disorders evolved.

Aim: This purpose of current study was to evaluate the outcomings of arthrocentesis combined with 
injection of hyaluronic acid in the treatment of inflammatory degenerative disease of temporomandibular joint.

\section{MATERIALS AND METHODS}

Eighty patients were included in this study (30 males and 50 females), age ranged from $18-55$ years. Those patients were examined and treated in the clinic of oral and maxillofacial surgery in Al-Wasity Teaching Hospital. Baghdad -Iraq, Ministry of Health. (from November 2015 to October 2017).

The inclusion criteria include:

1. The clinical signs.

A. pain at rest and chewing.

B. Mastication efficiency.

C. maximum mouth opening (MMO).

2. Previous known conservative management failed to resolve symptoms.

The exclusion criteria:

A. patient received chemo or radiotherapy.

B. patient with rheumatoid arthritis.

\section{Procedure}

The patients seated in supine position with skin of preauricular area were prepared. A refrance line (Holmlund-Hellsing line) was marked, eighteen gauge needles were inserted at 45 degree angle to corresponding plane during injection until reaching point space by feeling drop in the resistance to inserted needle. At least $120 \mathrm{ml}$ of $9 \%$ normal saline solution should be used to wash the joint out for optimum result during lavage. An ampoule of HA was connected to the needle in situ and $1 \mathrm{ml}$ of HA (Hyalgan, Fida, Albano, Italy) was injected into space. soft diet regimin was recommended for the patient . NSAID (Olfen* $100 \mathrm{mg}$, acino, Swiss) once daily was prescribed for 3 days with prophylactic antibiotic Augmentin $625 \mathrm{mg}$ three times daily ,see figure1.

\section{Follow up period}

Predetermined variables were assessed to test the efficacy of the treatment protocol. 4 months was the period between the 1st follow-up visit (at 1 month from 1st injection procedure) and 2nd follow up visit, all the parameters for TMJ functions measured with the same technique. The criteria for success was no pain VAS equal to zero, mastication efficiency was eating solid hard food.

*The evaluation between different follow up results was done by using monemar Chi square test and (Ftest) and the results were considered significant if $\mathrm{P}$ $<0.05$.

*The pain and Mastication efficiency data assessed by VAS from 0 to10.

* MMO data assessed by normal mouth opening range from $35-45 \mathrm{~mm}$

\section{RESULTS}

Eighty patients were included in this study, there were 50 females $(62.5 \%$ and 30 males $37.5 \%)$, with a mean age 31.5 years.

\section{Description and Statistics of Data \\ 1. Masticatory Efficiency}

The data obtained from visual analog value scale show significant reduction in masticatory efficiency from (6.75 to 2.25) at 5month follow up with (p value $<0.01$ ) with success rate $(87.5 \%)$. Pre-treatment and post-treatment data are shown in table (1).

Table 1 : Masticatory efficiency (before and after treatment).

\begin{tabular}{ccccc}
\hline & Pre-treatment & \multicolumn{2}{c}{ Post-treatment } & ANOVA \\
\cline { 2 - 5 } & No. $(\%)$ & $1^{\text {st }}$ follow-up visit & $2^{\text {nd }}$ follow-up visit \\
Masticatory Efficiency & $(1$ month $)$ & No. $(\%)$ & No. $(\%)$ & P-value \\
& & Total $80(100 \%)$ & Total 40 $(100 \%)$ & P $<0.01$ \\
& $80(100 \%)$ & Improved 40 $(50 \%)$ & Improved 30 $(75 \%)$ & HS $*$ \\
& Mean 6.75 & Mean 4.45 & Mean 2.25 & \\
& SD 0.168 & SD 0.111 & SD 0.056 & \\
\hline
\end{tabular}

\section{*Highly significant improvement in masticatory efficiency (p value $<0.01$ ).}

\section{Pain level}

The data optained from VAS revealed significant reduction in pain at 5 months follow up.

\section{A. Maximum pain at chewing}

In all patients maximum pain at chewing present and decrease in tendency. The pretreatment and post-treatment data are shown in table (2). 
Table 2: Maximum pain at chewing (before and after treatment).

\begin{tabular}{|c|c|c|c|c|}
\hline \multirow{4}{*}{ Maximum pain at chewing } & Pre-treatment & \multicolumn{2}{|c|}{ Post-treatment } & ANOVA \\
\hline & No. $(\%)$ & $\begin{array}{c}1^{\text {st }} \text { follow-up visit } \\
\text { (1 month) } \\
\text { No. }(\%)\end{array}$ & $\begin{array}{c}2^{\text {nd }} \text { follow-up visit } \\
\text { (4 month) } \\
\text { No. }(\%)\end{array}$ & P-value \\
\hline & $80(100 \%)$ & $\begin{array}{c}\text { Total } 80(100 \%) \\
\text { Improved } 40(50 \%)\end{array}$ & $\begin{array}{c}\text { Total } 40(100 \%) \\
\text { Improved } 30(75 \%)\end{array}$ & $\begin{array}{c}\mathrm{P}<0.01 \\
\mathrm{HS} *\end{array}$ \\
\hline & $\begin{array}{c}\text { Mean } 6.68 \\
\text { SD } 0.167\end{array}$ & $\begin{array}{c}\text { Mean } 3.53 \\
\text { SD } 0.088\end{array}$ & $\begin{array}{c}\text { Mean } 2.35 \\
\text { SD } 0.058\end{array}$ & \\
\hline
\end{tabular}

*Highly significant improvement in maximum pain at chewing ( $p$ value $<0.01$ ).

B. Maximum pain at rest

In all patients maximum pain at resting present and decrease in tendency. The data of pre-treatment and post-treatment are shown in table (3).

Table 3: Maximum pain at rest (before and after treatment).

\begin{tabular}{ccccc}
\hline & Pre-treatment & \multicolumn{2}{c}{ Post-treatment } & ANOVA \\
\cline { 2 - 5 } & No. $(\%)$ & $1^{\text {st }}$ follow-up visit & $2^{\text {nd }}$ follow-up visit & \\
Maximum pain at rest & $(1$ month $)$ & $(4$ month $)$ & P-value \\
& & No. $(\%)$ & No. $(\%)$ & Pol $)$ \\
& $80(100 \%)$ & Total $80(100 \%)$ & Total 40 $(100 \%)$ & P<0.01 \\
& Mean 5.50 & Mean 3.32 & Improved 30 (75\%) & HS * \\
& SD 0.137 & SD 0.083 & Mean 1.99 & \\
\hline
\end{tabular}

*Highly significant improvement in maximum pain at rest ( $\mathrm{p}$ value $<0.01$ ).

3. Maximal mouth opening

Initial measurement of maximum mouth opening detected 50 patients included in this study with a limited mouth opening. This study demonstrated success rate $(100 \%)$ with ( $P$ value $<\mathbf{0 . 0 1}$ ). As shown in table (4)

Table 4: Maximum mouth opening (before and after)

\begin{tabular}{|c|c|c|c|c|c|}
\hline \multirow{3}{*}{$\begin{array}{c}\text { mouth opening } \\
\text { evaluation }\end{array}$} & & Pre-treatment & $\begin{array}{c}1^{\text {st }} \text { follow up visit } \\
(1 \text { month })\end{array}$ & $\begin{array}{c}2^{\text {nd }} \text { follow up visit } \\
\text { (3 month) }\end{array}$ & P-value \\
\hline & No. & 50 & 50 & 50 & 0.003 \\
\hline & Mean & $32.5 \mathrm{~mm}$ & $36 \mathrm{~mm}$ & $40 \mathrm{~mm}$ & $\begin{array}{c}\mathrm{P}<0.01 \\
\mathrm{HS}^{*}\end{array}$ \\
\hline
\end{tabular}

*Highly significant improvement in MMO ( $\mathrm{p}<0.01$ value $)$.

\section{DISCUSSION}

The effectiveness of arthrocentesis procedure with HA injection in this study was based on 3 clinical parameters: Masticatory Efficiency, pain reduction during function and increase in MMO. Many clinicians and researchers have reported uniformly positive results of patients treated with arthrocentesis. All patients in the study were suffering from limited Masticatory Efficiency and their score equal to 6.75 rang from 5 to 8 . There is reduction in the mean of Masticatory Efficiency score to 4.45 ranged from 3 to 6 for all patients at 1st follow-up visit. Forty patients $50 \%$ showed complete response following single injection only, while remaining 40 patients $50 \%$ need 2 nd injection. The data illustrated highly significant reduction in Masticatory Efficiency from 6.75 to 2.25 with a $\mathrm{p}<$ 0.01 and a success rate of $87.5 \%$. These results agree with studies by D. Manfredini et al ${ }^{8}$. All patients in the study were complaining from TMJ pain, with a score of pain at mastication equal to 6.68 ranging from 5 to 8 and showed significant reduction in pain to 2.35 with a $\mathrm{p}<0.01$ with a success rate $87.5 \%$. These results agree with studies done by $D$. Manfredini et al (8). The data showed highly significant improvement in pain at rest from5.5 to 1.99 with a $p<0.01$ with a success rate $87.5 \%$. These results agree with studies done by $\mathrm{D}$. Manfredini et $\mathrm{al}^{(8)}$. The Study data showed significant improvement in maximum mouth opening of patients $\mathrm{P}<0.01$, with success rate $100 \%$. 
These results agree with studies done by Nitzan $\mathrm{DW}^{(9)}$ with the average mean for maximum mouth opening 32.5 to $40 \mathrm{~mm}$ with a $\mathrm{P}<0.01$.

\section{CONCLUSION}

The procedure of arthrocentesis with Sodium Hyaluronate injection offers therapeutic benefits, simplicity, safety, patient satisfaction, lack of significant side effects and complications.

\section{REFERENCES}

1. American Dental Association: Report of Presidents conference on the examination, diagnosis and management of temporomandibular disorders .J Am Dent Assoc 1988; 66:75.

2. Quinn JH, Bazan NG: Identification of prostaglandin E2 and leukotriene B4 in the synovial fluid of painful, dysfunction temporomandibular joint. J Oral Maxillofac Surg. 1990; 48:968.

3. Guarda-Nardini L, Stifano M, Brombin C et al. A one year series of arthrocentesis with hyaluronic acid injection for TMJ osteoarthritis. Oral surg Med Oral Pathol Oral Radiol Endod. 2007; 103:14-22.
4. Santos GS, Calado R, Sousa NE et al. Arthrocentesis Procedure:Using this therapeutic maneuver for TMJ closed lock Management: J Craniofac Surg. 2013; 24:1347-9.

5. Nitzan DW, Dolwick MF, Martinez GA. Temporomandibular Joint arthrocentesis; a simplified method for severe, limited mouth opening. J Oral Maxillofac Surg. 1991; 49:1163-1167.

6. Nitzan DW, Samson B, Better H. Long-term outcome of arthrocentesis for sudden-onset, persistent, severe closed lock of the Temporomandibular Joint. J Oral Maxillofac Surg. 1997; 55:151-157.

7. Peter A. Brennan Vellupillai Ilankovan, Arthrocentesis for TMJ pain Dysfunction syndrome. J Oral Maxillofac Surg. 2006; 64:949-951.

8. Manfredini D, Rancitelli D, Ferronato G, Guarda-nardini L. Arthrocentesis with or without additional drugs in Temporomandibular Joint inflammatory -degenerative disease: comparison of six protocol. J Oral Rehab. 2012; 39: $245-251$

9. Nitzan DW. Arthrocentesis for management of severe closed lock of the temporomandibular joint: current controversies in surgery for internal derangement of the temporomandibular joint. Atlas Oral Maxillofac Surg Clin North Am. 1994; 6: 245.
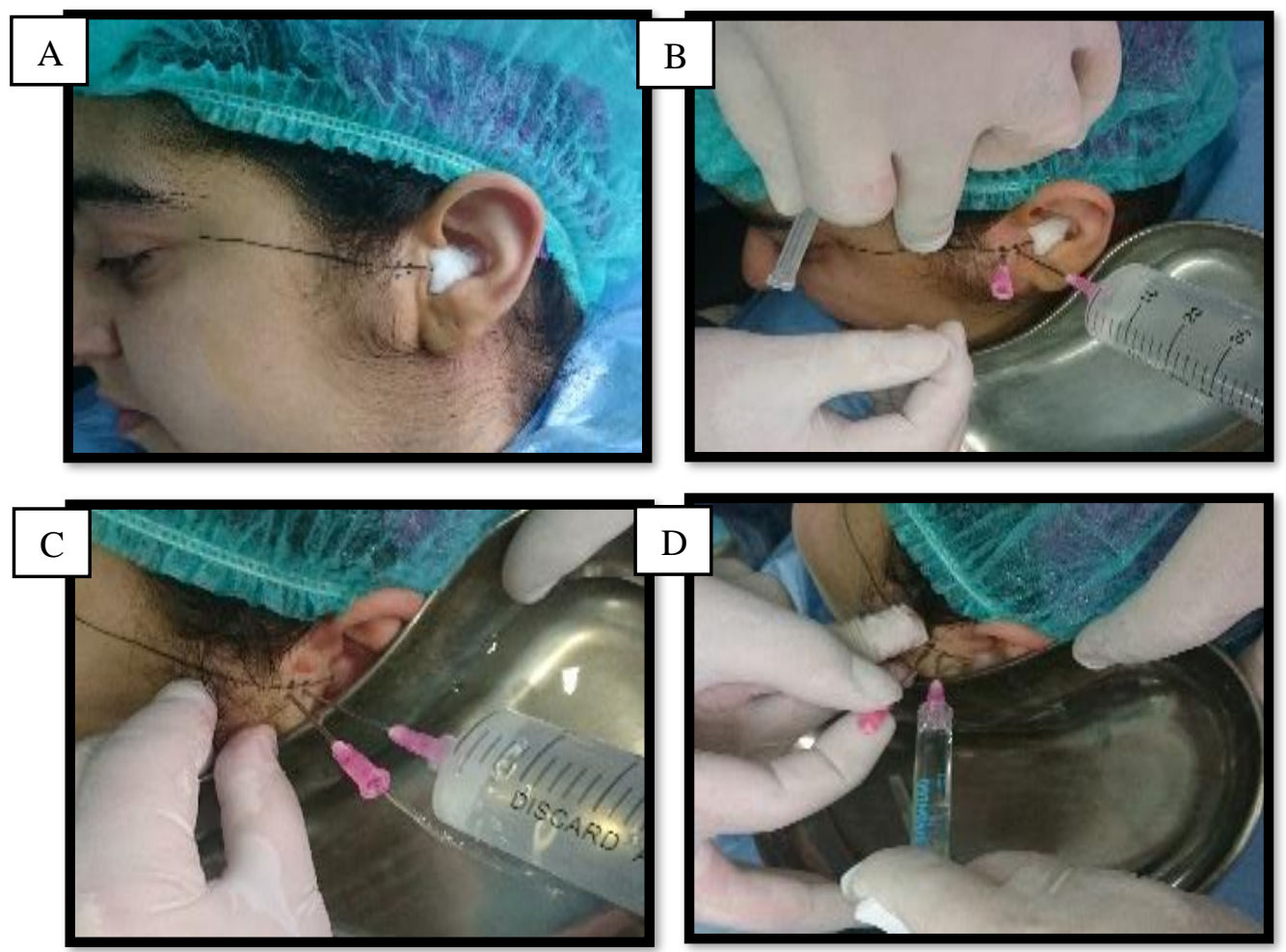

Figure 1: The manuver of arthrocentesis accompined by Hyaluronic Acid injection. (A) The drawn line from mid tragus of the ear to the lateral canthus of eye (Holmlund-Hellsing line), (B)The two needles procedure, (C)Wash with normal saline with spurt of solution from the second needle during the lavage, (D)Injection of $1 \mathrm{ml}$ of $\mathrm{HA}$. 


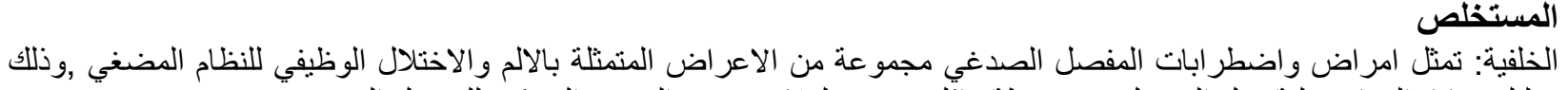

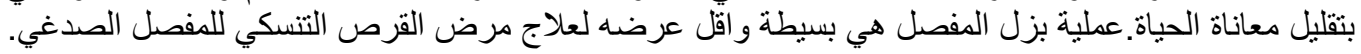

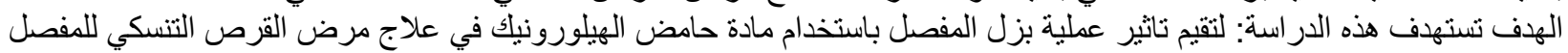
الصدغي

المادة والطريقة: ثمانون مريضا نم تتظيمهم خلال هذه الدراسة حيث يعاني الجميع من اعر اض واضطر ابات مرض القرص التنسكي

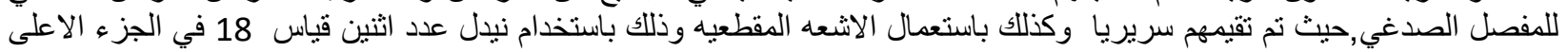

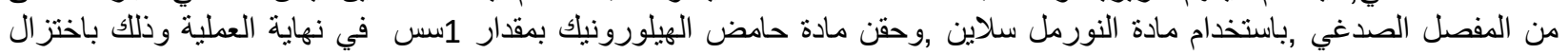

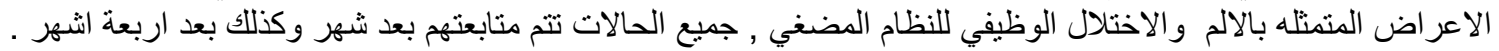

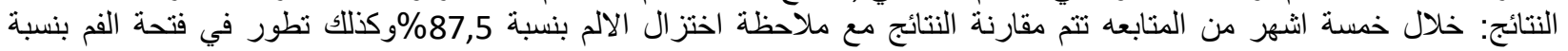

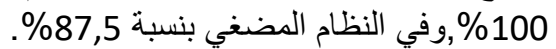

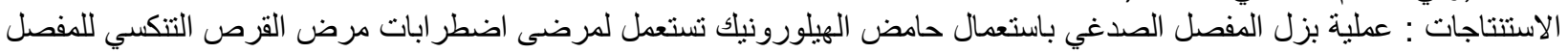

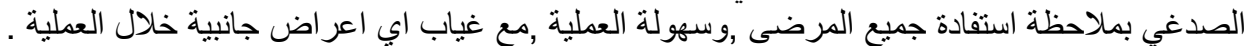

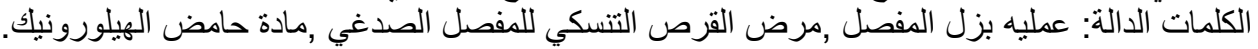

\section{(ब) (1)}

Articles Published by Journal of Baghdad College of Dentistry is licensed under a Creative Commons Attribution 4.0 International License. 